\title{
Association Between Active Gait Training for Severely Disabled Patients with Nasogastric Tube Feeding or Gastrostoma and Recovery of Oral Feeding: A Retrospective Cohort Study
}

This article was published in the following Dove Press journal:

Clinical Interventions in Aging

\author{
Hideki Arai $\mathbb{D}^{1,2}$ \\ Jiro Takeuchi DD $^{2}$ \\ Masafumi Nozoe ${ }^{3}$ \\ Tatsuyuki Fukuoka (iD) \\ Satoru Matsumoto' \\ Takeshi Morimoto (D) ${ }^{2}$ \\ 'Department of Rehabilitation, Toyonaka \\ Heisei Hospital, Toyonaka, Osaka, Japan; \\ ${ }^{2}$ Department of Clinical Epidemiology, \\ Hyogo College of Medicine, Nishinomiya, \\ Hyogo, Japan; ${ }^{3}$ Department of Physical \\ Therapy, Faculty of Nursing and \\ Rehabilitation, Konan Women's \\ University, Kobe, Hyogo, Japan; \\ ${ }^{4}$ Department of Rehabilitation, Faculty of \\ Rehabilitation, Hiroshima International \\ University, Higashihiroshima, Hiroshima, \\ Japan
}

Purpose: This study evaluates the effect of introducing active gait training (AGT) to patients who are severely disabled with nasogastric tube feeding or gastrostoma on the recovery of oral feeding.

Patients and Methods: We conducted a historical cohort study at a single rehabilitation center in Japan between January 2013 and December 2019. In this study, 154 severely disabled patients with nasogastric tube feeding or gastrostoma due to neurological diseases or disuse syndrome admitted in a rehabilitation ward were included, and their median age was 84 years. AGT was systematically implemented in August 2016, which consisted of using orthosis or assistance from physical therapists. We compared the recovery of oral feeding between periods before (Pre-AGT) and after (Post-AGT) the introduction of AGT.

Results: Among the 154 severely disabled patients included, 59 (38\%) were admitted in the Post-AGT period. Twenty-eight (30\%) and 54 patients (92\%) started gait training in the PreAGT and Post-AGT periods, respectively $(p<0.001)$. Significantly more patients recovered oral feeding in the Post-AGT than in the Pre-AGT periods (49\% vs $19 \%$, respectively; $p<$ 0.001). After the introduction of AGT, the adjusted hazard ratio for the recovery of oral feeding was 4.0 (95\% confidence interval, $1.9-8.3 ; p<0.001)$.

Conclusion: After the introduction of AGT to patients, increased recovery of oral feeding was observed in this retrospective evaluation. AGT should be considered for patients with tube feeding to help them recover oral feeding even if patients were severely disabled and required full assistance during gait training.

Keywords: active gait training, dysphagia, lateral hypothalamus, oral feeding, orexin, tube feeding

\section{Introduction}

A common and disabling condition after stroke is dysphagia, ${ }^{1}$ which is not influenced by the type of stroke. ${ }^{2}$ There is also no significant difference in the severity and characteristics of dysphagia between patients with stroke and those with traumatic brain injury. ${ }^{3}$ In addition, dysphagia is also present after disuse syndrome, ${ }^{4}$ which is a pathophysiological condition or symptom which affects body systems, such as the musculoskeletal, circulatory, and respiratory systems, which manifests as a result of prescribed or unavoidable inactivity. ${ }^{5}$
Correspondence: Takeshi Morimoto Department of Clinical Epidemiology, Hyogo College of Medicine, I-I, Mukogawa-cho, Nishinomiya-City, Hyogo 663-850I, Japan

Tel +8I-798-45-6III

Fax +8I-798-45-6920

Email morimoto@kuhp.kyoto-u.ac.jp 
Nasogastric tube feeding and gastrostoma are highly utilized to provide nutritional support for patients with swallowing difficulties. ${ }^{6}$ Patient populations most commonly receiving non-oral feeding support include patients with stroke $(65.1 \%)$ or dementia $(30 \%))^{7,8}$ However, nonoral feeding support did not exhibit a clear clinical benefit on patients with swallowing difficulties. ${ }^{8}$ For example, Finucane et al reported that non-oral feeding did not prevent aspiration pneumonia, prolong survival, improve wound healing, and reduce infections. ${ }^{8,9}$

The loss of body muscle mass or sarcopenia was associated with dysphagia. ${ }^{5}$ Therefore, interventions to increase muscle strength and early ambulation could be potential treatment options for patients with low nutrition status due to dysphagia. ${ }^{10}$ Yoshimura et al reported that chair-stand exercise improves post-stroke dysphagia. ${ }^{11}$ Besides, Takakusaki et al reported an association between locomotor function and feeding behavior. ${ }^{12,13}$ Since August 2016, we introduced active gait training (AGT) to patients who are severely disabled with nasogastric tube feeding or gastrostoma. Therefore, we conducted a historical cohort study to evaluate the relationship between the introduction of AGT to patients who are severely disabled with nasogastric tube feeding or gastrostoma and recovery of oral feeding.

\section{Methods}

\section{Study Design and Participants}

We conducted a historical cohort study at Toyonaka Heisei Hospital between January 2013 and December 2019. Patients admitted to the convalescent rehabilitation ward due to neurological diseases or disuse syndrome and those who were severely disabled and were not tolerable to independent gait training were considered eligible. However, those who could eat independently on admission were excluded from the study.

\section{Rehabilitation}

In August 2016, we introduced AGT to all of the severely disabled patients systematically for the improvement or prevention of disuse syndrome symptoms, including muscle atrophy, muscle weakness, contracture, cardiopulmonary disorders, deep vein thrombosis, and decubitus. The following types of AGT were implemented: gait training using orthosis and assisted gait training. We used different orthoses (eg, knee-ankle-foot orthosis, trunk-hip-bilateral knee-ankle-foot orthoses, and knee orthosis), which were some types of hospital equipment or were made to order, depending on the patients' conditions such as mildmoderate contracture of legs. In case of a patient requiring a mechanical ventilator, an attending physician assisted the physical therapist (PT) for gait training (Figure 1). Before July 2016, some patients received AGT based on the judgment of the PT in charge and attending physician. On the other hand, if the patients had moderate-severe contracture of legs and/or orthoses of hospital equipment did not suit them, then gait training was not conducted.

Throughout the study period, before and after the introduction of AGT, all patients received three 20-min sessions of physical, occupational, and speech language therapies every day (a total of nine sessions). The speech language therapist (ST) conducted indirect swallowing training for the patients' dysphagia. Oral sites such as the lower jaw, tongue, and cheeks were stimulated, and ice massage was performed to trigger the swallowing reflex. Video fluorography was used to assess the swallowing function based on the recommendations of the ST in charge before the introduction of AGT (Pre-AGT). Video fluorography was encouraged as a means to detect the slight changes in the swallowing function after the introduction of AGT (Post-AGT). The ST provided recommendations regarding direct swallowing training and oral intake based on clinical and/or instrumental swallowing assessment. Rehabilitation plan, alternative enteral nutrition (nasogastric tube feeding or gastrostoma), and

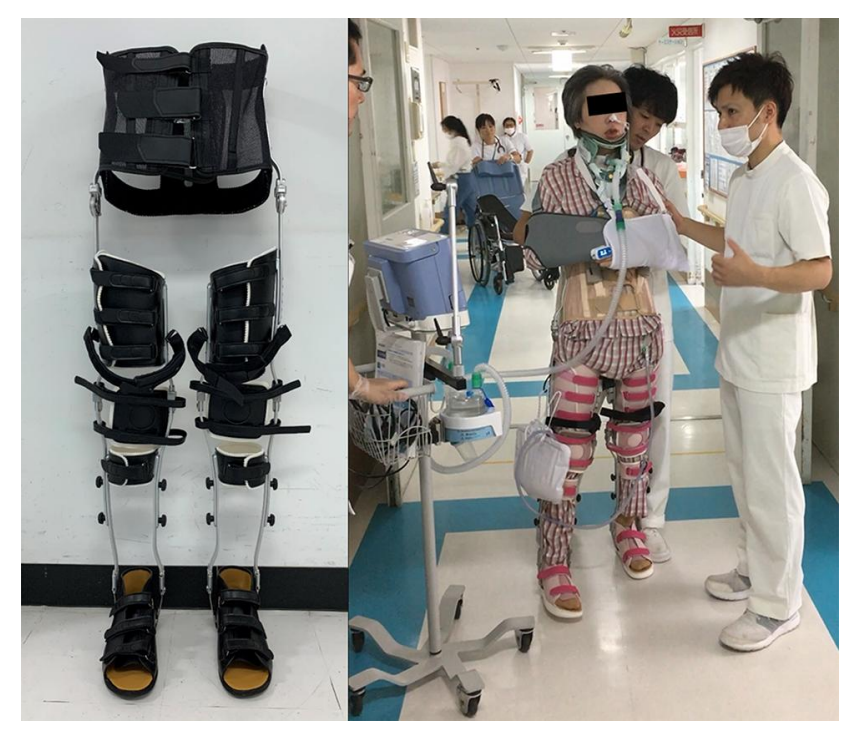

Figure I Trunk-hip-bilateral knee-ankle-foot orthoses and gait training used for a patient requiring tube feeding and mechanical ventilation.

Note: Informed consent was obtained from the people for publication of this image. 
intravenous fluids were discussed among the medical team consisting of an attending physician, nurse, PT, occupational therapist, ST, and nutritionist. If the changes in symptoms occurred, the ST reevaluated the dysphagia status and suggested whether the patients should transition from tube feeding to oral feeding.

\section{Measurements}

The following baseline clinical characteristics were evaluated: age, sex, body mass index, serum total protein, serum albumin, Glasgow coma scale, number of days of non-oral feeding before admission, feeding route, target disease for rehabilitation, video fluorography implementation, conducting gait training, recovery of oral feeding, number of days from admission to oral feeding, length of stay at convalescent rehabilitation, and number of patients who died or were transferred to other hospitals. We used the Functional Oral Intake Scale (FOIS) to measure the patients' oral intake status. The FOIS is a 7-point ordinal scale ( 1 , nothing by mouth; 2 , tube-dependent with minimal oral intake attempts of food or liquids; 3 , tubedependent with consistent oral intake of food or liquids; 4 , total oral diet of a single consistency; 5, total oral diet with multiple consistencies but requiring special preparations or compensations; 6 , total oral diet with multiple consistencies without special preparation but with specific food limitations; and 7, total oral diet with no restrictions). ${ }^{1,14}$ The attending physician and ST mainly discussed the decision of FOIS. Recovery of oral feeding was defined as the change from FOIS scores 1-3 on admission to FOIS scores 4-7 on discharge.

The Functional Independence Measure (FIM) was used to assess the patients' activities of daily living (ADLs). The FIM is a tool for the collection and comparison of rehabilitation outcomes, measurement of patients' progress, and planning treatment protocols. ${ }^{15}$ ADLs include self-care, eating, grooming, bathing, dressing, toileting, swallowing, sphincter control, mobility, transfer, and locomotion. ${ }^{15}$ The scale contains 18 items, of which 13 items are in the physical domains, and 5 items are related to cognition. ${ }^{15}$ Motor items measure self-care, sphincter control, locomotion, and transfer. ${ }^{15}$ Cognitive ones evaluate the subject's communication and social cognition. ${ }^{15}$ Based on the level of independence, each item is scored from 1 to 7 , where 1 indicates total dependence, and 7 represents complete independence. ${ }^{15}$ Possible total scores range from 18 to $126 .{ }^{15}$ Higher scores mean more independence in performing ADLs. ${ }^{15}$ The FIM score is indicative of the patients' level of disability and the burden of their care. ${ }^{15}$

\section{Statistical Analysis}

We present the baseline characteristics as median and interquartile range (IQR) for continuous variables and number and percentage for categorical variables. We compared the baseline characteristics and the outcome between the Pre-AGT and Post-AGT periods. We used the Wilcoxon rank-sum test for continuous variables and chisquare tests for categorical variables. Also, we used the Kaplan-Meier method to estimate the cumulative incidence of oral feeding. Using the Cox proportional hazard models, the effect of both Post-AGT and Pre-AGT on the recovery of oral feeding was estimated and expressed as hazard ratios (HRs) with 95\% confidence intervals (CIs). The HRs were adjusted for age, male, gastrostoma, neurological diseases, number of days of non-oral feeding before admission, FIM motor items at admission, and FIM cognitive items at admission. The continuous variables were included as continuous. We used the Wilcoxon signed-rank test to compare the FOIS and FIM scores between admission and discharge. All analyses were conducted using JMP 14.0 (SAS Institute Inc., Cary, NC, USA). Two-tailed $p$ values $<0.05$ were considered statistically significant.

\section{Ethical Considerations}

The study protocol complied with the World Medical Association's Declaration of Helsinki and the Ethical Guidelines for Medical and Health Research Involving Human Subjects issued by the Ministry of Health, Labor, and Welfare of Japan. The study was approved by the research ethics committee of Hyogo College of Medicine (no. 202,006-066). The requirement for a written informed consent was replaced by the opt-out method owing to the retrospective nature of this study.

\section{Result}

Of the 947 patients who were admitted during the study period, 793 were excluded because they presented with oral feeding on admission, and 154 were included in this study. Fifty-nine patients $(38 \%)$ were admitted in the PostAGT period (Figure 2). The number of days of non-oral feeding before admission was significantly higher in PreAGT period than in Post-AGT period (median 58 days vs 47 days, respectively; $p=0.01$ ). Significantly more patients with nasogastric tube feeding were present in the 


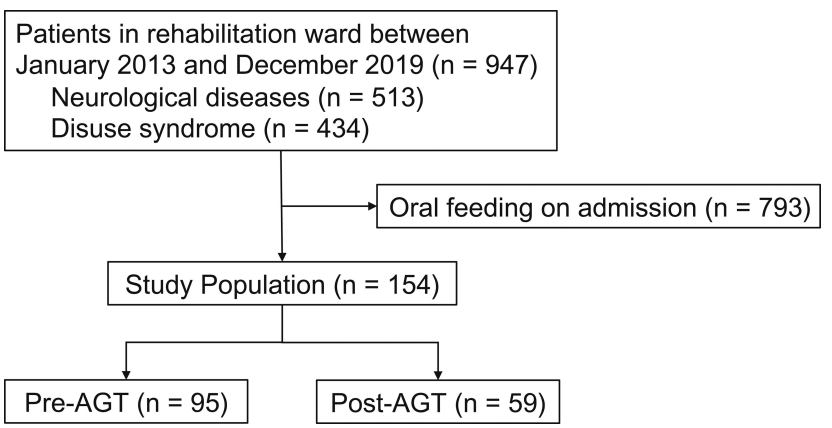

Figure 2 Study flow diagram.

Abbreviation: AGT, active gait training.

Post-AGT period than in the Pre-AGT period $(93 \%$ vs $47 \%$, respectively; $p<0.001)$. Significantly more patients with neurological diseases were present in the Post-AGT period than in the Pre-AGT period (73\% vs $53 \%$, respectively; $p=0.02$ ). The FOIS scores at admission between the Pre-AGT and Post-AGT periods were not significantly different (median 1 vs 1 , respectively; $p=0.07$ ). The FIM scores at admission between the Pre-AGT and Post-AGT periods were not significantly different (median 20 vs 20, respectively; $p=0.3$ ). Significantly video fluorography was conducted more in Post-AGT period than in PreAGT period ( $86 \%$ vs $10 \%$, respectively; $p<0.001)$ (Table 1). Twenty-eight (30\%) and 54 patients (92\%) started gait training in the pre-AGT and post-AGT periods, respectively ( $p<0.001)$. Significantly more patients transitioned to oral feeding in the Post-AGT period than in the Pre-AGT period (49\% vs 19\%, respectively; $p<0.001$ ). The number of days from admission to oral feeding was significantly lower in Post-AGT period than in Pre-AGT period (median 19 days vs 55 days, respectively; $p=$ 0.002 ) (Table 2). The cumulative incidence of oral feeding at 270 days was $31.7 \%$ in Pre-AGT period and $58.6 \%$ in Post-AGT period (log-rank $p<0.001$ ) (Figure 3). The adjusted HR of recovery of oral feeding in Post-AGT period was 4.0 (95\% CI, 1.9-8.3; $p<0.001)$ (Table 3). Significant differences were found in the FOIS scores at discharge between the Pre-AGT and Post-AGT periods (median 1 vs 4, respectively; $p<0.001$ ). Except for the patients who died or were transferred to other hospitals, the FIM scores at discharge between the Pre-AGT and Post-AGT periods were not significantly different (median 23 vs 26 , respectively; $p=0.1$ ) (Table 4 ).

Regarding the way of conducting gait training, no significant difference between Pre-AGT period and PostAGT period was found (Table 5). The most frequent
Table I Baseline Clinical Characteristics

\begin{tabular}{|c|c|c|c|}
\hline \multirow[t]{2}{*}{ Characteristics } & \multicolumn{2}{|l|}{ No. (\%) } & \multirow[t]{2}{*}{$P$ value } \\
\hline & $\begin{array}{l}\text { Pre-AGT } \\
(\mathrm{n}=95)\end{array}$ & $\begin{array}{l}\text { Post-AGT } \\
(n=59)\end{array}$ & \\
\hline Age, year, median (IQR) & $83(76-90)$ & $84(77-90)$ & 0.9 \\
\hline Male & $36(38)$ & $34(58)$ & 0.02 \\
\hline $\begin{array}{l}\text { Body mass index, } \mathrm{kg} / \mathrm{m}^{2} \text {, } \\
\text { median (IQR) }\end{array}$ & $18(16-20)$ & $19(17-22)$ & 0.02 \\
\hline $\begin{array}{l}\text { Total protein, g/dl, median } \\
\text { (IQR) }\end{array}$ & $6.5(6.1-6.8)$ & $6.3(6.0-6.6)$ & 0.06 \\
\hline Albumin, g/dl, median (IQR) & $3.5(3.1-3.7)$ & $3.5(3.3-3.8)$ & 0.4 \\
\hline $\begin{array}{l}\text { Glasgow coma scale, } \\
\text { median (IQR) }\end{array}$ & II (9-15) & II (9-14) & 0.4 \\
\hline $\begin{array}{l}\text { Number of days of non-oral } \\
\text { feeding before admission, } \\
\text { median (IQR) }\end{array}$ & $58(46-85)$ & $47(36-62)$ & 0.01 \\
\hline \multicolumn{4}{|l|}{ Feeding tube } \\
\hline Gastrostoma & $50(53)$ & $4(7)$ & $<0.001$ \\
\hline Nasogastric tube & $45(47)$ & $55(93)$ & \\
\hline \multicolumn{4}{|l|}{$\begin{array}{l}\text { Target disease for } \\
\text { rehabilitation }\end{array}$} \\
\hline Disuse syndrome & $45(47)$ & $16(27)$ & 0.02 \\
\hline Neurological diseases & $50(53)$ & $43(73)$ & \\
\hline Ischemic stroke & $28(30)$ & $25(42)$ & \\
\hline Hemorrhagic stroke & $19(20)$ & $13(22)$ & \\
\hline Traumatic brain injury & $3(3)$ & $3(5)$ & \\
\hline Neuromuscular disease & $0(0)$ & $2(3)$ & \\
\hline FOIS, median (IQR) & $I(1-1)$ & $I(I-I)$ & 0.07 \\
\hline \multicolumn{4}{|l|}{ FIM, median (IQR) } \\
\hline Total & $20(18-28)$ & $20(18-26)$ & 0.3 \\
\hline Motor items & $13(13-15)$ & $13(13-14)$ & 0.08 \\
\hline Cognitive items & $7(5-11)$ & $6(5-11)$ & 0.7 \\
\hline Video fluorography & $9(10)$ & $51(86)$ & $<0.001$ \\
\hline
\end{tabular}

Abbreviations: FOIS, Functional Oral Intake Scale; FIM, Functional Independence Measure.

reason of non-gait training was moderate-severe contracture of legs $(n=29,40 \%)$. Although all causes of non-gait training in the Post-AGT period were identified, in the PreAGT period, the reasons of non-gait training for more than half of the patients were unknown (Table 6).

\section{Discussion}

The introduction of AGT to patients who are severely disabled with feeding tubes increased the recovery of oral feeding. Additionally, after the introduction of AGT, the number of days from admission to oral feeding was decreased. This study is the first to retrospectively evaluate the association between AGT and the recovery of oral feeding. 
Table 2 Clinical Outcomes Before and After the Introduction of Active Gait Training

\begin{tabular}{|c|c|c|c|}
\hline \multirow[t]{2}{*}{ Characteristics } & \multicolumn{2}{|l|}{ No. (\%) } & \multirow[t]{2}{*}{$P$ value } \\
\hline & $\begin{array}{l}\text { Pre-AGT } \\
(n=95)\end{array}$ & $\begin{array}{l}\text { Post-AGT } \\
(n=59)\end{array}$ & \\
\hline Gait training & $28(30)$ & $54(92)$ & $<0.001$ \\
\hline Recovery of oral feeding & $18(19)$ & $29(49)$ & $<0.001$ \\
\hline $\begin{array}{l}\text { Number of days from } \\
\text { admission to oral } \\
\text { feeding, median (IQR) }\end{array}$ & $55(45-127)$ & $19(12-57)$ & 0.002 \\
\hline $\begin{array}{l}\text { Length of stay at } \\
\text { convalescent } \\
\text { rehabilitation, days, } \\
\text { median (IQR) }\end{array}$ & $114(64-177)$ & $|5|(90-177)$ & 0.2 \\
\hline Death & $12(13)$ & $5(9)$ & 0.6 \\
\hline $\begin{array}{l}\text { Transfer to other } \\
\text { hospitals }\end{array}$ & $5(5)$ & $4(7)$ & 0.7 \\
\hline
\end{tabular}

Abbreviation: AGT, active gait training.

The association of AGT and the recovery of oral feeding was not well investigated from pathophysiological perspectives, but several hypotheses were suggested. Orexin was a hormone associated with not only locomotor but also feeding behavior. ${ }^{12,13}$ Orexin A and Orexin B are synthesized in the lateral hypothalamic and perifornical areas. ${ }^{13,16}$ Orexin A and B are excitatory multitasking neuropeptides regulating a set of vital body functions, including locomotion, feeding and appetite behavior, central regulation of gut function, sleep/wake rhythms, maintenance of wakefulness, energy homeostasis, thermoregulation, reward system, spontaneous physical activity, cognition and mood regulation, cardiovascular responses, and activating activities of a sympathetic nerve. ${ }^{12,13,16}$ Orexin neurons do not only produce orexins $\mathrm{A}$ and $\mathrm{B}$ but also are directly and indirectly activated by orexin. ${ }^{17}$ Orexins $\mathrm{A}$ and $\mathrm{B}$ neurons are restricted to the lateral and posterior areas of the hypothalamus; whereas, both orexins A and B nerve fibers project widely into the olfactory bulb, cerebral cortex, thalamus, hypothalamus, and brainstem. ${ }^{18}$ The extensive distribution of orexins suggests that they function as neuromodulators and/or neurotransmitters in a wide variety of neural circuitry. ${ }^{18}$ In addition, physical exercise increases the level of orexin in the plasma of humans. ${ }^{16}$ As described above, AGT could regulate the feeding behavior of patients who are severe through orexin hormone actions.

Damage to the left superior frontal gyrus, left dorsal anterior cingulate gyrus, left hypothalamus, and left nucleus accumbens was a significant predictor of FOIS score changes in patients with stroke. ${ }^{1}$ These regions mainly comprise structures of the limbic system (eg, cingulate gyrus and hypothalamus) or are connected to the limbic system (eg, nucleus accumbens). ${ }^{1}$ These structures are known to be involved in emotions, motivation, and reward functions, as well as hunger and thirst regulation. ${ }^{1}$ Orexin neurons involved in locomotion and feeding behavior exist in the lateral hypothalamic area. ${ }^{12,13,16}$ Therefore, AGT could regulate the feeding behavior of severe patients by stimulating the limbic system.

In this study, the HR of oral feeding for patients with neurological diseases was not statistically significant compared to those with disuse syndrome. Although there is no report which compares the improvement of dysphagia between patients with neurological disease and disuse syndrome, it is clear that disuse syndrome had a negative impact on the improvement of swallowing function. ${ }^{4}$ Besides the disuse syndrome, the period from the onset of severe dysphagia to the beginning of swallowing training could be an important factor to the prognosis. ${ }^{4}$ Despite the fact that the number of days of non-oral feeding before admission was significantly higher in Pre-AGT period than in Post-AGT period, a similar tendency to oral feeding was seen among patients in Pre-AGT and Post-AGT periods. Additionally, low FIM and cognitive dysfunction affect the outcome of dysphagia. ${ }^{2,4}$ Although the FIM motor item and FIM cognitive item scores at admission in both PreAGT and Post-AGT periods were low, we found that significantly more patients transitioned to oral feeding in the Post-AGT period than in the Pre-AGT period. In addition, male patients were significantly less likely to recover oral feeding than female patients. These findings were consistent with those found by Nakajima et al who reported that, at 3 months after stroke onset, male sex had a negative impact on oral intake. ${ }^{19}$

In this study, the HR for gastrostoma was also not statistically significant compared to nasogastric tube feeding. Significantly, it is clear that more patients with nasogastric tube feeding were present in the PostAGT period than in the Pre-AGT period. Prolonged nasogastric tube placement exhibits a negative impact on the patient's swallowing function which includes higher functional dysphagia scale scores, which mean worse swallowing function, slowing of the pharyngeal transit time, abnormal epiglottic movement pattern, residues in the valleculae and pyriform, increased penetration-aspiration rating, and edema in the pharynx and/or larynx. ${ }^{20}$ Therefore, nasogastric tube feeding is more 


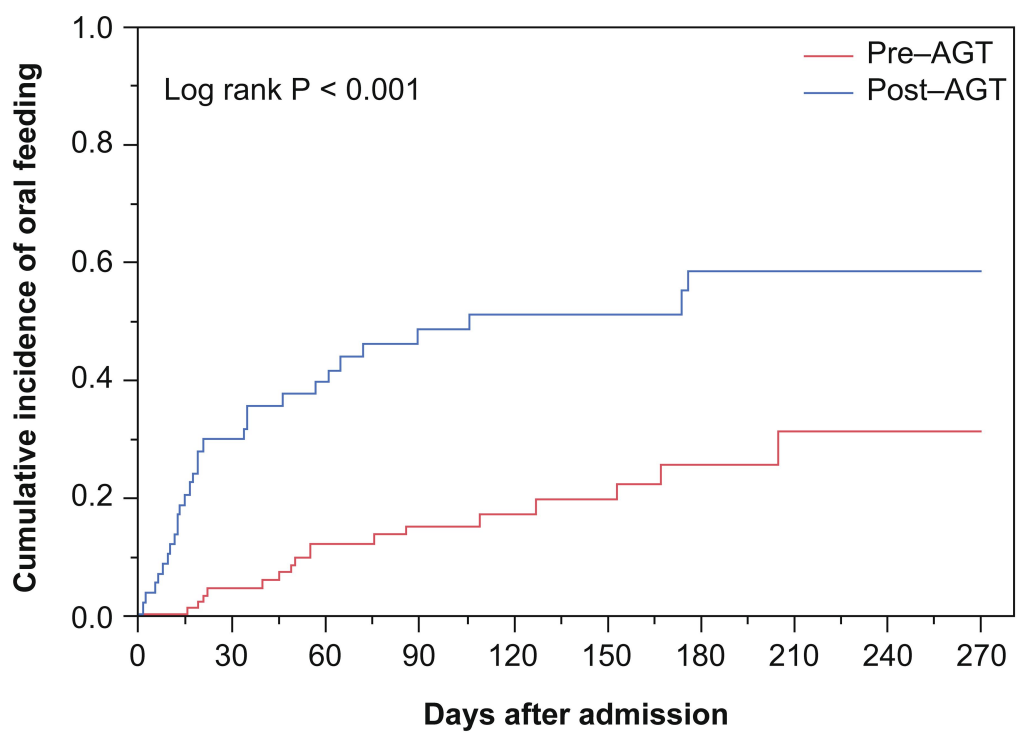

\begin{tabular}{|c|c|c|c|c|}
\hline Days after admission & 0 & 90 & 180 & 270 \\
\hline \multicolumn{5}{|l|}{ Pre-AGT } \\
\hline Cumulative incidence & & $15.4 \%$ & $25.4 \%$ & $31.7 \%$ \\
\hline Number of cumulative cases of oral feeding & & 12 & 16 & 17 \\
\hline Number of cohorts & 95 & 48 & 17 & 2 \\
\hline \multicolumn{5}{|l|}{ Post-AGT } \\
\hline Cumulative incidence & & $48.5 \%$ & $58.6 \%$ & $58.6 \%$ \\
\hline Number of cumulative cases of oral feeding & & 26 & 29 & 29 \\
\hline Number of cohorts & 59 & 20 & 4 & 1 \\
\hline
\end{tabular}

Figure 3 Cumulative incidence of oral feeding.

Abbreviation: AGT, active gait training.

disadvantageous than gastrostomy tube feeding. AGT period. This suggests that treating patients with Nevertheless, significantly more patients transitioned to nasogastric tubes is more effective than treating them oral feeding in the Post-AGT period than in the Pre- with PEG immediately because research showed that

Table 3 Factors Associated with the Recovery of Oral Feeding

\begin{tabular}{|c|c|c|c|}
\hline \multirow[t]{2}{*}{ Variables } & \multirow{2}{*}{$\begin{array}{l}\text { Crude } \\
\text { HR }(95 \% \mathrm{Cl})\end{array}$} & \multicolumn{2}{|l|}{ Adjusted* } \\
\hline & & HR (95\% Cl) & $P$ value \\
\hline Post-AGT & $3.3(1.8-6.0)$ & $4.0(1.9-8.3)$ & $<0.001$ \\
\hline Age & $0.97(0.95-0.99)$ & $0.98(0.96-1.0)$ & 0.1 \\
\hline Male & $0.8(0.4-1.4)$ & $0.4(0.2-0.8)$ & 0.006 \\
\hline Gastrostoma & $0.2(0.09-0.6)$ & $0.7(0.2-2.3)$ & 0.5 \\
\hline Neurological diseases & $1.6(0.8-3.2)$ & $0.9(0.4-2.0)$ & 0.8 \\
\hline Number of days of non-oral feeding before admission & $0.99(0.98-0.99)$ & $0.99(0.98-1.0)$ & 0.2 \\
\hline FIM motor items at admission & $1.0(0.98-I . I)$ & I.I (0.98-I.I) & 0.2 \\
\hline FIM cognitive items at admission & I.I (I.0-I.I) & $1.0(0.96-I .1)$ & 0.4 \\
\hline
\end{tabular}

Note: *Adjusted HR was calculated from the Cox proportional hazard model with all listed variables.

Abbreviations: HR, hazard ratio; $\mathrm{Cl}$, confidence interval; AGT, active gait training; FIM, Functional Independent Measure. 
Table 4 Comparison of Functional Oral Intake Scale and Functional Independence Measure Scores

\begin{tabular}{|c|c|c|c|c|c|c|c|}
\hline \multirow[t]{2}{*}{ Variables } & \multicolumn{3}{|c|}{ Pre-AGT $(n=78)$} & \multicolumn{3}{|c|}{ Post-AGT $(n=50)$} & \multirow[t]{2}{*}{$P$ value $f$} \\
\hline & Admission & Discharge & $P$ value* & Admission & Discharge & $P$ value* & \\
\hline FOIS, median (IQR) & $I(I-I)$ & $I(I-3)$ & $<0.001$ & $I(I-I)$ & $4(I-5)$ & $<0.001$ & $<0.001$ \\
\hline \multicolumn{8}{|l|}{ FIM, median (IQR) } \\
\hline Total & $21(18-27)$ & $23(18-35)$ & $<0.001$ & $20(18-25)$ & $26(20-45)$ & $<0.001$ & $<0.001$ \\
\hline Motor items & $13(13-15)$ & $15(|3-2|)$ & $<0.001$ & $13(13-14)$ & $15(13-29)$ & $<0.001$ & 0.02 \\
\hline Cognitive items & $7(5-11)$ & $8(5-13)$ & $<0.001$ & $6(5-10)$ & $9(6-20)$ & $<0.001$ & $<0.001$ \\
\hline
\end{tabular}

Notes: *P value for change from admission to discharge within group. $\dagger P$ value for difference of changes from admission to discharge between groups.

Abbreviations: AGT, active gait training; FOIS, Functional Oral Intake Scale; FIM, Functional Independence Measure.

Table 5 Details of Active Gait Training

\begin{tabular}{|c|c|c|c|}
\hline & \multicolumn{2}{|l|}{ No. (\%) } & \multirow[t]{2}{*}{$P$ value } \\
\hline & $\begin{array}{l}\text { Pre-AGT } \\
(n=28)\end{array}$ & $\begin{array}{l}\text { Post-AGT } \\
(n=54)\end{array}$ & \\
\hline \multicolumn{4}{|l|}{ Orthosis } \\
\hline Knee-ankle-foot orthosis & $6(2 I)$ & $13(24)$ & 0.1 \\
\hline $\begin{array}{l}\text { Trunk-hip-bilateral knee- } \\
\text { ankle-foot orthoses }\end{array}$ & I (4) & $14(26)$ & 0.2 \\
\hline $\begin{array}{l}\text { Bilateral knee-ankle-foot } \\
\text { orthosis }\end{array}$ & I (4) & $12(22)$ & 0.4 \\
\hline Knee orthosis & I (4) & $0(0)$ & 0.2 \\
\hline Assisted gait & II (39) & $6(11)$ & 0.5 \\
\hline Walking assistance device & $7(25)$ & $9(17)$ & 0.3 \\
\hline T-cane & I (4) & $0(0)$ & 1.0 \\
\hline
\end{tabular}

Table 6 Reason of Withholding Active Gait Training

\begin{tabular}{|l|l|l|l|}
\hline \multicolumn{1}{|c|}{} & \multicolumn{2}{|l|}{ No. (\%) } & \multirow{2}{*}{ P value } \\
\cline { 2 - 3 } & $\begin{array}{l}\text { Pre-AGT } \\
(\mathbf{n}=\mathbf{6 7})\end{array}$ & $\begin{array}{l}\text { Post-AGT } \\
(\mathbf{n}=\mathbf{5})\end{array}$ & \\
\hline $\begin{array}{l}\text { Contracture of legs } \\
\text { Active infection }\end{array}$ & $28(42)$ & $\mathrm{I}(20)$ & 0.6 \\
$\begin{array}{l}\text { Orthostatic } \\
\text { hypotension }\end{array}$ & $2(0)$ & $3(60)$ & $<0.00 \mathrm{I}$ \\
Bone fracture & $0(0)$ & 1.0 \\
Cancer pain & $\mathrm{I}(\mathrm{I})$ & $\mathrm{I}(20)$ & 0.07 \\
Unknown & $36(54)$ & $0(0)$ & 1.0 \\
$0(0)$ & 0.054 \\
\hline
\end{tabular}

gait and swallowing training can effectively help patients transition to oral feeding.

This study exhibits several limitations. First, we did not assess the swallowing ability of our patients during speech therapy. Therefore, the effect of swallowing difficulty on the findings in this study was uncertain. Although we adjusted the potential confounders, we excluded the swallow function, and some other important confounders might be incorporated. Thus, our findings could be different when the assessment of swallowing function was conducted. Second, we did not consider the distance in and frequency of the gait training. Although we assessed whether gait training was conducted for patients who were severely disabled, we did not examine the details of the gait training. The relationship between the total load and recovery of oral feeding should be evaluated in the future. Third, this study was a pre-post comparison between Pre-AGT and Post-AGT which was not robust. However, a multivariable Cox proportional hazard model was used to adjust potential confounders to alleviate the systematic differences in these confounders. Finally, generalizing the results of this study should be cautious because this was a single-center study.

\section{Conclusion}

After the introduction of AGT, increased recovery of oral feeding was observed in this retrospective evaluation. Moreover, AGT might increase oral feeding and such recovery of oral feeding should lead to improved quality of life which leads to patients' dignity. Therefore, AGT should be considered for patients with tube feeding to facilitate the probability to regain oral feeding even if patients were severely disabled and required full assistance during gait training.

\section{Funding}

This work was not supported by any grant.

\section{Disclosure}

The authors report no conflicts of interest.

\section{References}

1. Wilmskoetter J, Bonilha L, Martin-Harris B, et al. Factors influencing oral intake improvement and feeding tube dependency in patients with poststroke dysphagia. J Stroke Cerebrovasc Dis. 2019;28(6):14 21-1430. doi:10.1016/j.jstrokecerebrovasdis.2019.03.031 
2. Falsetti P, Acciai C, Palilla R, et al. Oropharyngeal dysphagia after stroke: incidence, diagnosis, and clinical predictors in patients admitted to a neurorehabilitation unit. J Stroke Cerebrovasc Dis. 2009;18(5):329-335. doi:10.1016/j.jstrokecerebrovasdis.2009.01.009

3. Lee WK, Yeom J, Lee WH, et al. Characteristics of dysphagia in severe traumatic brain injury patients: a comparison with stroke patients. Ann Rehabil Med. 2016;40(3):432-439. doi:10.5535/arm.20 16.40.3.432

4. Kojima A, Imoto Y, Osawa Y, et al. Predictor of rehabilitation outcome for dysphagia. Auris Nasus Larynx. 2014;41(3):294-298. doi:10.1016/j.anl.2013.12.009

5. Fujishima I, Fujiu-Kurachi M, Arai H, et al. Sarcopenia and dysphagia: position paper by four professional organizations. Geriatr Gerontol Int. 2019;19(2):91-97. doi:10.1111/ggi.13591

6. Gomes CAR Jr, Andriolo RB, Bennett C, et al. Percutaneous endoscopic gastrostomy versus nasogastric tube feeding for adults with swallowing disturbances. Cochrane Database Syst Rev. 2015;5:1-76.

7. Wirth R, Volkert D, Bayer JM, et al. PEG tube placement in german geriatric wards - retrospective data-base analysis. $Z$ Gerontol Geriatr. 2007;40(1):21-30.

8. Livia S, Aarthi M, Giselle C, et al. Dysphagia in the elderly: management and nutritional considerations. Clin Interv Aging. 2012; 7:287-298.

9. Finucane TE, Christmas C, Travis K. Tube Feeding in patients with advanced dementia: a review of the evidence. JAMA. 1999;282 (14):1365-1370. doi:10.1001/jama.282.14.1365

10. Wakabayashi H. Presbyphagia and sarcopenic dysphagia: association between aging, sarcopenia, and deglutition. J Frailty Aging. 2014;3 (2):97-103.

11. Yoshimura Y, Wakabayashi H, Nagano F, et al. Chair-stand exercise improves post-stroke dysphagia. Geriatr Gerontol Int. 2020;1-7.
12. Takakusaki K, Takahashi K, Saitoh K, et al. Orexinergic projections to the cat midbrain mediate alternation of emotional behavioural states from locomotion to cataplexy. J Physiol. 2005;568(3): 1003-1020.

13. Okumura T, Takakusaki K. Role of orexin in central regulation of gastrointestinal functions. $J$ Gastroenterol. 2008;43(9):652-660. doi:10.1007/s00535-008-2218-1

14. Yi YG, Shin H-I. Psychometrics of the functional oral intake scale for infants. Front Pediatr. 2019;7:1-8. doi:10.3389/fped.2019.00156

15. Rayegani SM, Raeissadat SA, Alikhani E, et al. Evaluation of complete functional status of patients with stroke by functional independence measure scale on admission, discharge, and six months poststroke. Iran J Neurol. 2016;15(4):202-208.

16. Sergio C, Marco C, Vincenzo M, et al. Orexin system: the key for a healthy life. Front Physiol. 2017;31:1-9.

17. Yamanaka A, Tabuchi S, Tsunematsu T, et al. Orexin directly excites orexin neurons through orexin 2 receptor. $J$ Neurosci. 2010;30 (38):12642-12652. doi:10.1523/JNEUROSCI.2120-10.2010

18. Date Y, Ueta Y, Yamashita H, et al. Orexins, orexigenic hypothalamic peptides, interact with autonomic, neuroendocrine and neuroregulatory systems. Proc Natl Acad Sci. 1999;96(2):748-753. doi:10.1073/ pnas.96.2.748

19. Nakajima M, Inatomi Y, Yonehara T, et al. Temporal trends in oral intake ability 3 months after acute ischaemic stroke: analysis of a single-centre database from 2003 to 2011. J Rehabil Med. 2014;46 (3):200-205. doi:10.2340/16501977-1260

20. Wang Z-Y, Chen J-M, Ni G-X. Effect of an indwelling nasogastric tube on swallowing function in elderly post-stroke dysphagia patients with long-term nasal feeding. BMC Neurol. 2019;19(1):1-7. doi:10.1186/s12883-019-1314-6
Clinical Interventions in Aging

\section{Publish your work in this journal}

Clinical Interventions in Aging is an international, peer-reviewed journal focusing on evidence-based reports on the value or lack thereof of treatments intended to prevent or delay the onset of maladaptive correlates of aging in human beings. This journal is indexed on PubMed Central, MedLine, CAS, Scopus and the Elsevier
Bibliographic databases. The manuscript management system is completely online and includes a very quick and fair peer-review system, which is all easy to use. Visit http://www.dovepress.com/ testimonials.php to read real quotes from published authors. 